\title{
Formação de professores em robótica educacional com Hardware Livre Arduino no contexto Um Computador por Aluno ${ }^{1}$
}

\author{
Marcos de Castro Pinto, Marcos da Fonseca Elia, Fábio Ferrentini Sampaio \\ Núcleo de Computação Eletrônica - Universidade Federal do Rio de Janeiro (UFRJ) \\ Caixa Postal 2324 - CEP 21.941-590 - Rio de Janeiro - RJ - Brasil \\ marcastp@gmail.com, melia@nce.ufrj.br,ffs@nce.ufrj.br
}

\begin{abstract}
In nowadays we deal daily with technological artifacts that enable us to communicate, work, fun, etc. All the time we are learning and relearning to operate all these artifacts, in an endless process of teaching-learning. Robotics in education contributes to bring the school to this "technological world", stimulating and motivating students and teachers in the construction of knowledge in an interdisciplinary way. The present work aims to present the experience gained in the application of course for teacher training in educational Robotics, using interactive peer to peer teaching model and the Arduino open source hardware, in two schools participating in the government program PROUCA: a computer per student in the borough of Pirai, Rio de Janeiro State.
\end{abstract}

Resumo. Lidamos cotidianamente com artefatos tecnológicos que nos possibilitam comunicar, trabalhar, divertir, etc. A todo o momento estamos aprendendo e reaprendendo a operar todos estes artefatos, em um interminável processo de ensino-aprendizagem. A robótica aplicada na educação contribui em aproximar a escola deste "mundo" tecnológico, estimulando e motivando alunos e professores na construção de saberes nas mais variadas áreas do conhecimento. O presente trabalho tem o objetivo de apresentar a experiência obtida na aplicação de curso para formação de professores em robótica educacional, utilizando modelo pedagógico interativo e o hardware livre Arduino. em duas escolas participantes do programa governamental PROUCA: Um Computador por Aluno no município de Piraí, Rio de Janeiro.

\section{Introdução}

Estamos diante de um mundo dinâmico, em uma constante transformação fomentada pelas inovações tecnológicas em diversas áreas do conhecimento. Esta nova dinâmica, balizada, principalmente, por Tecnologias de Informação e Comunicação (TIC) constitui a base da moderna sociedade da informação impactando diretamente no dia-a-dia das pessoas. Neste contexto, as escolas brasileiras tem sido levadas a reboque e não como sujeito desta nova dinâmica mundial. Políticas públicas recentes têm tentado reverter

\footnotetext{
${ }^{1}$ Este trabalho foi financiado com recursos do edital CNPq/CAPES/SEED-MEC n ${ }^{\circ}$ 76/2010
} 
este quadro, destacando-se o programa "Um Computador por Aluno (PROUCA)", iniciado em 2007 com o objetivo de "Promover a inclusão digital nas escolas das redes públicas de ensino estadual, distrital ou municipal, mediante a aquisição de computadores portáteis novos, com conteúdos pedagógicos, destinados ao desenvolvimento dos processos de ensino-aprendizagem" (BNDES/PROUCA, 2012).

A fase pré-piloto do PROUCA contemplou cinco escolas públicas nos municípios de São Paulo/SP, Porto Alegre/RS, Palmas/TO, Piraí/RJ e Brasília/DF. Atualmente, o programa encontra-se na fase II (piloto), sendo aplicada em 300 escolas públicas, distribuídas por todos os estados da federação.

Com o objetivo de incentivar projetos de desenvolvimento científico, tecnológico e inovação vinculados a fase II do PROUCA, foi lançado ao final de 2010 o edital $\mathrm{CNPq} / \mathrm{CAPES} / \mathrm{SEED}-\mathrm{MEC} \mathrm{n}^{\mathrm{o}} 76 / 2010$. Dentre os projetos selecionados no referido edital, encontra-se o projeto do grupo GINAPE/UFRJ intitulado "Uca na Cuca", visando ações voltadas para as três modalidades previstas no edital: pesquisa científica, pesquisa tecnológica e inovação pedagógica, elegendo como tema problematizador a Robótica Educacional.

A robótica aplicada na educação, conhecida como robótica educacional ou robótica pedagógica, colabora em muito para uma prática pedagógica instigadora, motivadora da aprendizagem, principalmente pelo fato de exercitar o manuseio físico de diversos dispositivos e equipamentos (FILHO, 2008). Para Schons et al. (2004), a robótica pedagógica "constitui nova ferramenta que se encontra à disposição do professor, por meio da qual é possível demonstrar na prática muitos dos conceitos teóricos, às vezes de difícil compreensão, motivando tanto o professor como principalmente o aluno". A robótica contribui ainda para o desenvolvimento do raciocínio lógico e para a união de conhecimentos teóricos e experimentação prática (MIRANDA, 2006). Outro ponto que merece destaque na aplicação da robótica em educação diz respeito a possibilidade de discutir temas que transversalizam diferentes áreas do conhecimento (CÉSAR, 2007), possibilitando a integração de diferentes componentes curriculares.

O presente artigo está organizado em seis seções, incluindo esta Introdução. Na seção 2 são apresentados duas iniciativas de hardware livre para aplicação em robótica educacional, com foco no projeto Arduino. A seção 3 aborda a necessidade de capacitação de docentes em robótica educacional. A organização do curso de robótica com hardware livre Arduino é apresentada na seção 4, bem como o modelo pedagógico interativo orientador do curso. Na seção 5 são apresentados os resultados obtidos na aplicação do curso com professores do município de Piraí. Finalmente, na seção 6 são apresentadas nossas considerações finais.

\section{O Hardware Livre Arduino}

As possibilidades para utilização da robótica no contexto educacional ampliam-se fortemente com a incorporação das tecnologias livres, seja de software ou de hardware, tanto em função do acesso aos recursos tecnológicos envolvidos por qualquer pessoa interessada quanto pelos custos normalmente mais baixos que permeiam projetos com estas tecnologias. Além disso, projetos com tecnologias livres estão associados a internet e a comunidade de adeptos que se auxiliam mutuamente no sentido de 
esclarecimento, soluções de problemas e fortalecimento dos projetos. Neste sentido, destacamos duas iniciativas de harware livre com aplicações em robótica na educação: a placa eletrônica programável GoGo Board, desenvolvida no Future of Learning Group do MIT (Massachusetts Institute of Technology) para projetos de robôs e dispositivos de interação homem-computador e o projeto Arduino, criado na Itália em 2005 com o objetivo de oferecer uma plataforma de prototipagem eletrônica (placa eletrônica programável) de baixo custo e de fácil manuseio por qualquer pessoa interessada em criar projetos com objetos e ambientes interativos (ARDUINO, 2012). A GoGo Board e o Arduino (figura 1) caracterizam-se como projetos de hardware "open source", onde a documentação para elaboração da placa eletrônica é disponibilizada para os usuários em seus sites de projeto. O projeto Arduino disponibiliza para download o ambiente de desenvolvimento $\left(\mathrm{IDE}^{2}\right)$ para a programação da placa eletrônica, fornecendo ainda o código fonte deste ambiente. O IDE Arduino pode rodar nas plataformas Windows e Linux e possui uma linguagem de programação baseada no projeto Wiring ${ }^{3}$.
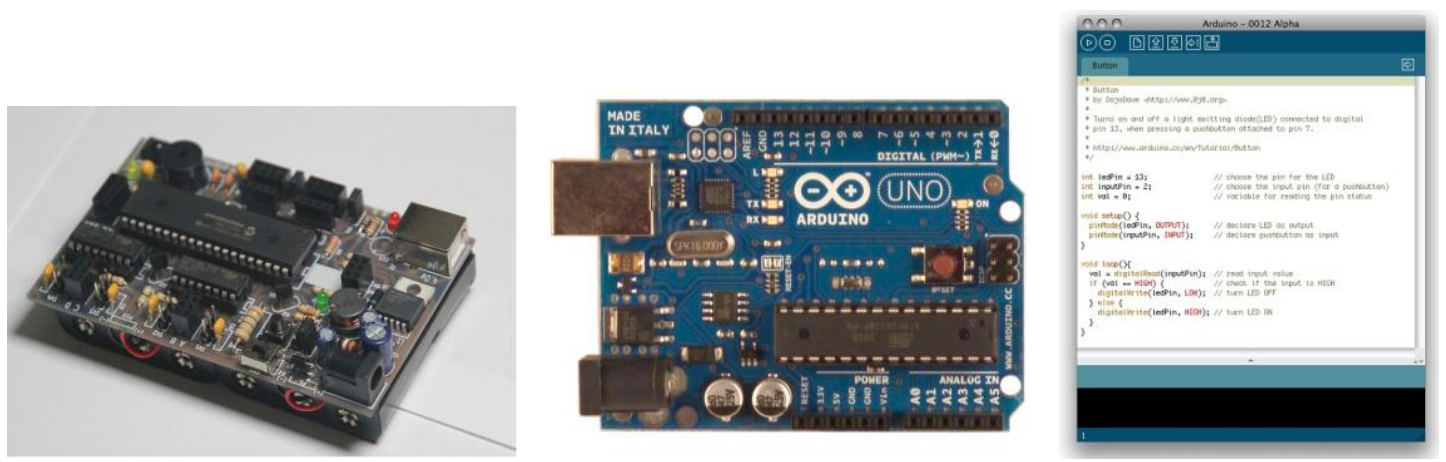

Figura 1 - Placa GoGo Board, Placa Arduino e IDE Arduino

Além do ambiente padrão para programação (no contexto procedural) disponibilizado no site do projeto, encontramos hoje na web alguns projetos de ambientes gráficos para programação no Arduino, entre elas, destacamos o S4A-Scratch for Arduino, o Ardublock e o Minibloq ${ }^{4}$. O projeto Uca na Cuca do grupo GINAPE/UFRJ possui como uma de suas metas (meta 4) a adaptação da linguagem icônica "Programe fácil" (MIRANDA, 2006) para uso com o Arduino e computadores (classmates) do PROUCA.

A plataforma Arduino tem se popularizado ao redor do mundo em uma infinidade de aplicações, possibilitando que pessoas não especialistas em eletrônica e programação possam colocar em prática suas idéias de interação com objetos e ambientes fazendo uso de recursos da eletrônica e da programação. A grande popularidade do projeto incentivou diversas empresas a fabricarem o Arduino a custos bastante acessíveis, inclusive no Brasil (uma placa Arduino montada pode ser encontrada no mercado brasileiro por volta dos $\mathrm{R} \$ 100,00)$. Nesse contexto, o Arduino torna-se uma excelente opção para aplicação em robótica educacional nas escolas brasileiras, seja pelo baixo custo, seja pela possibilidade de funcionamento em software livre.

\footnotetext{
${ }^{2}$ IDE - Integrated Development Environment (Ambiente de Desenvolvimento Integrado)

3 http://www.wiring.org.co/

${ }^{4}$ Scratch for Arduino: http://seaside.citilab.eu/scratch/arduino/, Ardublock: http://blog.ardublock.com, Minibloq: http://blog.minibloq.org/
} 


\section{Formação de professores em robótica educacional (problematização)}

Para que os recursos tecnológicos se tornem aliados efetivos ao trabalho docente, faz-se necessário um sólido e contínuo processo de capacitação de professores para que estes sintam-se efetivamente capazes para utilizar as tecnologias de modo crítico (CURCIO, 2008, pág. 34). Segundo Kenski,

(...) é preciso que este profissional tenha tempo e oportunidades de familiarização com as novas tecnologias educativas, suas possibilidades e limites para que, na prática, faça escolhas conscientes sobre o uso das formas mais adequadas ao ensino de um determinado tipo de conhecimento, em um determinado nível de complexidade, para um grupo específico de alunos e no tempo disponível. (KENSKI, 1998)

No contexto da robótica educacional, é de grande importância o papel do professor atuando tanto no planejamento quanto na execução de sua atividade didática com os recursos da robótica, agindo como elemento mediador e incentivador para que seus alunos obtenham êxito em suas tarefas propostas. Para tal, torna-se necessário que o professor sinta-se capacitado a trabalhar com tecnologias que envolvam a robótica educacional.

O projeto Uca na Cuca contempla em uma das metas (meta 1) a capacitação de docentes no campo da robótica educacional, aliado a tecnologias de baixo custo que possibilitem a inserção da robótica no cotidiano das escolas públicas. Como resultado desta ação, foi desenvolvido o "Curso de Robótica Educacional com Hardware Livre Arduino", com o objetivo de sensibilizar professores de escolas participantes do Projeto PROUCA para o uso da robótica educacional como elemento motivador da aprendizagem de seus alunos. $\mathrm{O}$ referido curso teve como base a pesquisa de dissertação de mestrado "Aplicação de arquitetura pedagógica na formação de professores em Robótica educacional com componentes de baixo custo" (PINTO, 2011), desenvolvida no PPGI/UFRJ.

\section{Curso de robótica educacional com Hardware Livre Arduino}

O curso possui dois eixos de orientação: pedagógico e tecnológico; conforme ilustrado pela rede sistêmica da figura 2 .

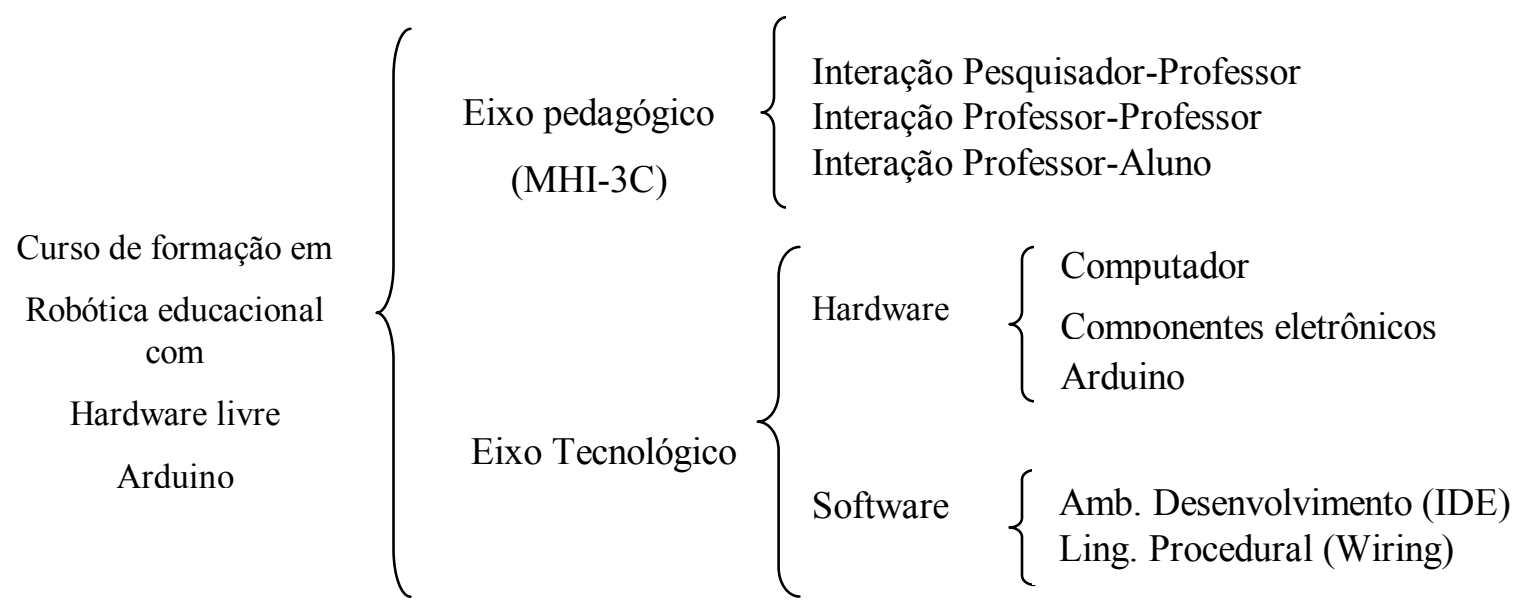

Figura 2 - Rede Sistêmica do Curso de Robótica Educacional com Arduino 
O eixo pedagógico propõe uma arquitetura pedagógica que deve propiciar um ambiente favorável para autoria coletiva de atividades didáticas (PINTO, 2011) e interativo com os diversos atores no curso. Esta arquitetura, mostrada na figura 3, é denominada de Modelo Hierárquico de Interatividade em três camadas (MHI-3C).

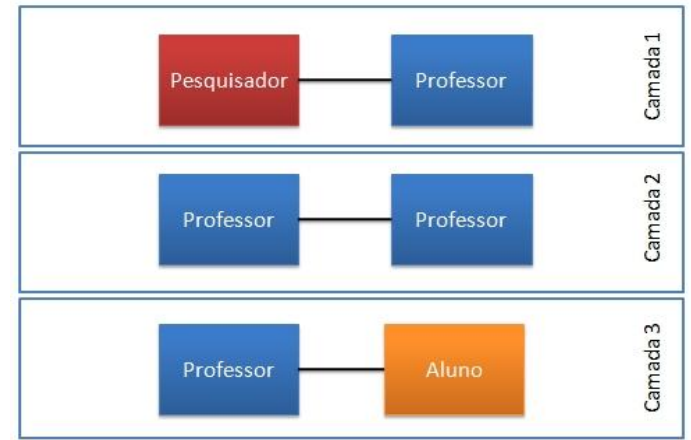

Figura 3 - Modelo Hierárquico de Interatividade em três camadas

$\mathrm{Na}$ primeira camada pesquisadores e professores do projeto participam de discussões em fóruns virtuais para orientações quanto à proposta do projeto. Esta discussão teórica é permeada com instâncias práticas, propostas pela equipe de pesquisadores do projeto, que ajudem a ilustrar e a compreender os conceitos em tela. No segundo nível (camada 2), professores discutem entre si propostas de atividades didáticas no contexto do projeto e as constroem. Em seguida (camada 3), os professores aplicam as atividades didáticas junto aos seu alunos, ou seja, discutem com os seus alunos o que construir dentro de um projeto pedagógico previamente desenhado por eles. É importante ressaltar que mesmo nas camadas 2 e 3 permanece a participação da equipe de pesquisadores do projeto, só que de maneira cada vez mais discreta.

O eixo tecnológico do curso envolve o uso do hardware, como a placa eletrônica Arduino, computadores para programação da placa e diversos componentes eletrônicos para as atividades com robótica. Além do hardware, este eixo do curso abrangia o software de programação para o Arduino (IDE) e a linguagem para programação Arduino, de característica procedural, baseado no projeto Wiring.

O curso foi proposto no formato semipresencial com um número máximo de 16 professores (integrantes das escolas-piloto do programa PROUCA e parceiras no projeto Uca na Cuca) e organizado em três fases de acordo com o diagrama de atividades mostrado na figura 4.

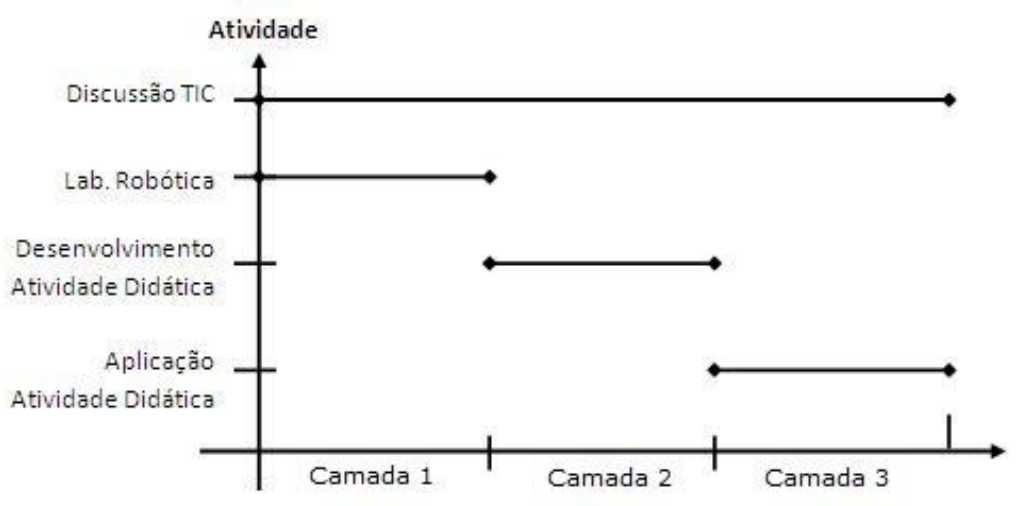

Figura 4 - Diagrama de atividades do curso 
Ao final do curso, os professores cursitas devem apresentar um relatório final descrevendo como foi a aplicação da atividade em sala de aula e qual sua avaliação sobre a experiência realizada.

\section{Aplicação do curso (Experiência com professores do município de Piraí)}

A primeira turma do curso de robótica educacional com hardware livre Arduino do projeto Uca na Cuca foi formada com professores oriundos das duas escolas parceiras do projeto, localizadas no município de Piraí (RJ). São elas: Escola Municipal Lúcio de Mendonça e Escola Municipal CIEP 477 Prof $^{a}$ Rosa da Conceição Guedes (esta última é também integrante do PROUCA/MEC). A Secretaria de Educação de Piraí possui atualmente 21 escolas onde todos os alunos da rede utilizam laptops educacionais.

O curso, com duração de aproximadamente três meses, foi aplicado entre fevereiro e maio de 2012. A turma foi inicialmente composta de dez professores, sendo cinco oriundos da disciplina de Matemática, quatro de Ciências e um de História. Do total de professores, sete concluíram o curso com êxito. É importante ressaltar que os professores da turma não tinham experiência anterior com robótica. As expectativas dos professores em relação ao curso puderam ser observadas nos depoimentos postados no fórum "Apresentação da Turma" presente no ambiente virtual de aprendizagem utilizado no curso (plataforma Moodle do NCE/UFRJ) ${ }^{5}$. Apresentamos abaixo um destes depoimentos.

"(...) leciono para o ensino médio no estado e estou atuando como coordenadora de Matemática na E.M. Lúcio de Mendonça, onde já lecionei em sala de aula por 11 anos. Estou muito entusiasmada com este curso de Robótica e espero poder aprender muitas coisas novas para aplicá-las com os alunos.Podem contar comigo!!!!" (Professora da EM Lúcio de Mendonça)

Seguindo o modelo hierárquico de interatividade apresentado neste artigo, a primeira camada de interação (entre pesquisadores e professores) tinha como objetivo principal fundamentar conceitos relacionados com tecnologias aplicadas à educação e com a robótica educacional. Nesse sentido, foram disponibilizados no ambiente de aprendizagem alguns textos e vídeos com temas relacionados aos conceitos propostos, bem como dois fóruns virtuais para troca de idéias sobres estes temas.

O primeiro fórum postado teve o seguinte título "O professor no contexto das inovações tecnológicas" e tinha como objetivo discutir o papel do professor neste novo contexto onde as inovações tecnológicas permeiam todas as classes sociais e por todos os setores da sociedade, entre eles, a escola. O segundo fórum, estreitamente ligado ao escopo do curso, possuiu o seguinte título: "A Róbótica e a sala de aula". A proposta deste fórum era discutir sobre o uso da robótica no contexto educacional, pensando tanto nos aspectos positivos para o processo ensino-aprendizagem, quanto em pontos negativos que dificultam a implantação e disseminação de práticas pedagógicas com robótica.

Além das interações à distância através do ambiente virtual de aprendizagem, esta primeira camada do curso contemplou três encontros presenciais destinados as oficinas de robótica. Estas oficinas tiveram o objetivo de ambientação e familiarização dos

\footnotetext{
${ }^{5}$ Disponível no endereço: http://ava.nce.ufrj.br/
} 
professores com os diversos componentes e materiais usados em robótica. Os encontros presenciais aconteceram no Laboratório de Robótica de Piraí, localizado no pólo $\mathrm{CEDERJ}^{6}$ do município. No primeiro encontro, foram apresentados diversos componentes eletro-eletrônicos tais como pilhas, baterias, resistores, matriz de contatos, LED`s, motores DC, etc. Foi disponibilizado no ambiente virtual de aprendizagem um arquivo com informações básicas sobre estes principais componentes eletro-eletrônicos.

$\mathrm{O}$ segundo e terceiro encontros presenciais foram destinados à apresentação da plataforma Arduino e de práticas com programas exemplos. Para execução das práticas, foram fornecidos kits contendo fios, pilhas, componentes eletro-eletrônicos, além da placa eletrônica Arduino. O ambiente de desenvolvimento (IDE) para o Arduino foi instalado pela equipe GINAPE/UFRJ nos quatro laptops educacionais (classmates) do laboratório de robótica, os quais possuíam o sistema operacional Meego, fornecido pela empresa Metasys. A figura 5 mostra um classmate conectado a uma placa Arduino e professores utilizando o mesmo para programação com o ambiente Arduino.
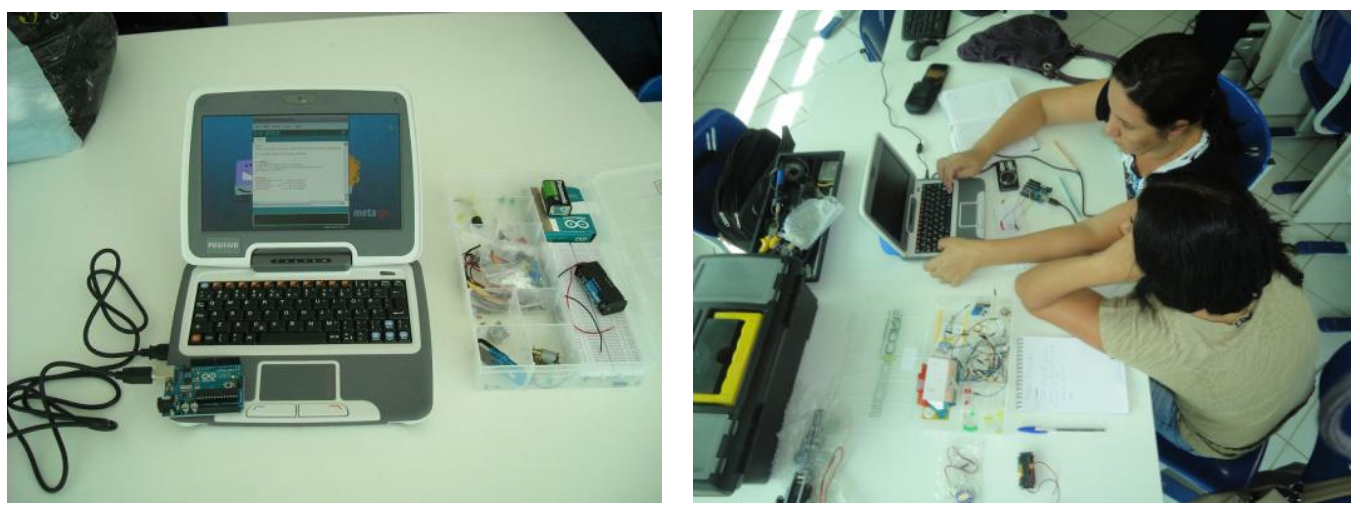

Figura 5 - (a) Classmate com So Meego e Arduino (b) Professores programando com o Arduino

$\mathrm{Na}$ segunda fase (camada) do curso, os professores interagiram entre si discutindo sobre os conteúdos ligados a robótica educacional abordados na primeira camada. A turma foi dividida em grupos onde cada grupo deveria entregar uma proposta de atividade didática com robótica, segundo um roteiro pré-estabelecido pelo pesquisador. $\mathrm{O}$ quadro 1 apresenta os grupos formados com as atividades didáticas propostas.

Quadro 1 - Atividades didáticas propostas pelos grupos

\begin{tabular}{|c|c|c|}
\hline \multicolumn{3}{|c|}{ Proposta de atividade didática com robótica educacional } \\
\hline Grupo & Nome da atividade & Disciplinas envolvidas \\
\hline Primavera & Número, LED's ${ }^{7}$ e Reta Real & Matemática \\
\hline Verão & Integrobótica & Matemática, Ciências, História \\
\hline
\end{tabular}

\footnotetext{
${ }^{6}$ CEDERJ - Consórcio de Universidades públicas presentes no Estado do Rio de janeiro com o objetivo de oferecer educação superior na modalidade a distância (EAD). O consórcio é formado pelas seguintes instituições: UENF, UERJ, UFF, UFRJ, UFRRJ e UNIRIO.
}

${ }^{7}$ LED (Light Emitting Diode) - Diodo emissor de luz 
Houve ainda ao final nesta camada um encontro presencial com a equipe de pesquisadores para suporte aos professores na utilização dos componentes eletroeletrônicos e da plataforma Arduino para implementação das atividades propostas.

A terceira e última fase do curso culminou com a aplicação das atividades didáticas com robótica junto aos alunos da escolas piloto do projeto. O grupo Primavera aplicou sua proposta com alunos do $9^{\circ}$ ano da EM Lúcio de Mendonça. A proposta do grupo (Número, LED's e Reta Real) tinha como objetivo o estudo dos números racionais e sua representação na reta real utilizando a robótica como elemento lúdico para estímulo ao aprendizado. Para tal, foi elaborado pelos professores um jogo formado por uma caixa com LED's na parte superior dispostos em forma de reta (cada LED possuía uma cor indicava um ponto na reta) e cartões contendo diversos números racionais (figura 6). A caixa com LED's possuía ainda uma fenda para inserção do cartão com a resposta escolhida e um botão para acionamento de um dos LED's da reta. Basicamente, o jogo possuía a seguinte dinâmica: um grupo de alunos apertava o botão para acionar apenas um dos LED na reta (escolha aleatória). De acordo com a cor do LED acionado, os alunos pegavam a ficha-resposta com o número racional que consideravam correto e a introduziam na fenda presente na caixa. Esta, com a função de um leitor formado por um LED como fonte de luz e um sensor $\mathrm{LDR}^{8}$, acusava se a resposta estava certa ou errada através de mensagem apresentada no monitor serial do IDE Arduino. A confecção do jogo e a programação do Arduino foi realizada pelos professores do grupo.

O trabalho proposto pelo grupo Verão (Integrobótica) foi aplicado com alunos do $9^{\circ}$ ano da EM Prof ${ }^{a}$ Rosa da Conceição Guedes CIEP 477. Este trabalho tinha uma característica multidisciplinar visando a integração das disciplinas Matemática, Ciências e História. O trabalho consistiu na elaboração de um mapa do Brasil constituído de LED's que indicavam o desmatamento da Mata Atlântica ao longo dos séculos após a chegada dos Portugueses em terras brasileiras. O mapa possuía ainda outro conjunto de LED's formando um gráfico que indicava o percentual de desmatamento ao longo dos séculos (figura 6). Segundo relato dos professores do grupo Verão, os alunos participaram ativamente na confecção do mapa, na montagem dos LED's e na programação do Arduino.

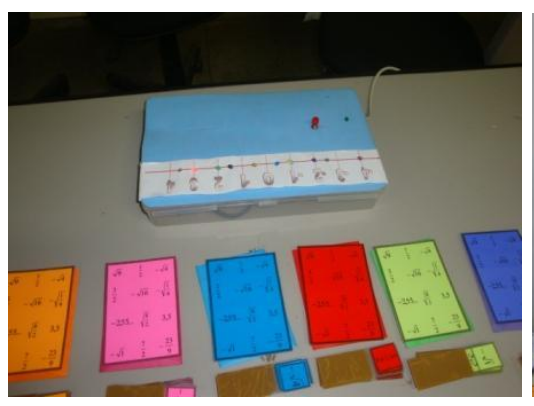

Figura 6 - (a) Jogo Grupo Primavera

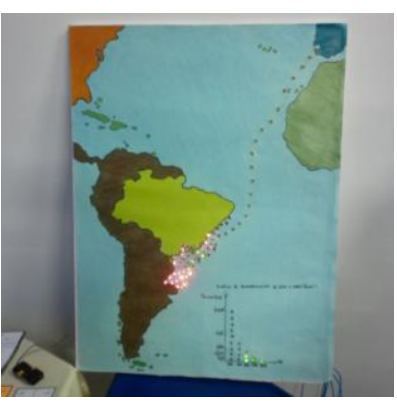

(b) Mapa Grupo Verão Arduino

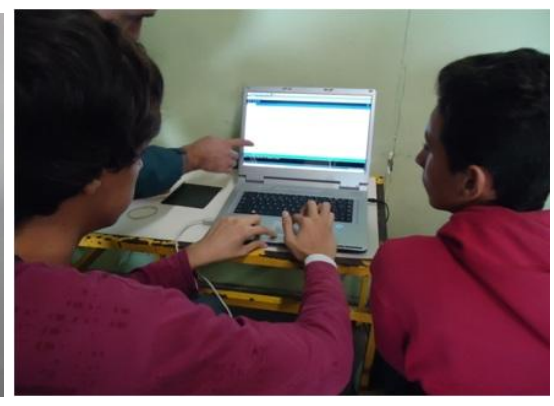

(c) Alunos programando o

Como evento de encerramento do curso, ocorreu o último encontro presencial onde os dois grupos realizaram uma apresentação sobre suas experiências com a aplicação das atividades didáticas com robótica para a equipe do projeto Uca na Cuca.

\footnotetext{
${ }^{8}$ LDR (Light Dependent Resistor) - Resistor dependente de luz
} 
Neste evento estavam presentes também representantes da Secretaria de Educação do município de Piraí.

\section{Considerações Finais}

Acredita-se que o curso proposto neste projeto tenha colaborado de forma significativa para a inserção da robótica educacional no cotidiano dos professores participantes. A utilização do hardware livre Arduino no curso foi importante para que os professores tomassem ciência sobre possibilidades de recursos para robótica educacional, acessíveis tanto em custo como em complexidade de programação. A união entre recursos tecnológicos e baixo custo tornam o Arduino uma excelente opção para composição de kits de robótica para uso em escolas públicas.

O modelo estruturado de interação aplicado no curso (modelo MHI-3C) contribuiu para um caráter dinâmico com participação ativa dos professores nas três camadas de interação. Sobretudo, a última camada (Professor-Aluno) colaborou sobremaneira para multiplicação e continuidade do processo de forma autossustentável (apropriação da proposta). Nesta direção, cinco professores desta primeira turma submeteram um projeto, denominado REP (Robótica e Educação em Piraí), para a Secretaria de Educação de Piraí com o objetivo de aplicação da robótica de forma continuada nas escolas municipais Lúcio de Mendonça e Prof Rosa da Conceição Guedes. Ainda sobre multiplicação e continuidade, é importante comentar que os resultados positivos alcançados pelos professores em suas experiências com a robótica (e com o Arduino) contribuíram para despertar o interesse de outros professores sobre o tema. Como resultado, está em andamento a segunda turma do curso de robótica educacional com Hardware Livre Arduino, envolvendo professores de outras escolas da rede municipal de Piraí. Este efeito multiplicador, balizado principalmente pelo fato de resultar das experiências dos próprios professores, torna-se relevante para consolidação da proposta do projeto Uca na Cuca em sua meta de capacitar professores em robótica na Educação no contexto "Um Computador por Aluno".

Por último, torna-se importante ressaltar a satisfação dos professores com a motivação e o "encantamento" dos alunos nas atividades com robótica. Esta afirmativa é reforçada pelo depoimento de uma das professoras em sua apresentação ao final do curso.

"Eles deram uma resposta para nós que eu fiquei encantada mesmo... Vocês vão ver pelas fotos... Foi muito legal... O Olhinho de cada um daqueles alunos ali, foi muito gratificante... " (Professora da EM Lúcio de Mendonça)

\section{Agradecimentos}

Agradecemos o apoio da Secretaria de Educação de Piraí pelo transporte e estrutura ofertada aos professores do curso e aos mestrandos do PPGI/UFRJ Armando Luiz e Rafael Machado pela preciosa colaboração nos encontros presenciais. 


\section{Referencias}

ARDUINO. Arduino. Disponível em http://www.arduino.cc/ . Acesso em julho de 2012.

BNDES/PROUCA.Disponível em:

http://www.bndes.gov.br/SiteBNDES/bndes/bndes_pt/Institucional/Apoio_Financeiro/Pro gramas_e_Fundos/prouca.html. Acesso em julho de 2012.

CÉSAR, D. R. ; BONILLA, M. H. S. Robótica livre: implementação de um ambiente dinâmico de robótica pedagógica com soluções tecnológicas livres no Cet CEFET em Itabirito - Minas Gerais - Brasil. WORKSHOP SOBRE INFORMÁTICA NA ESCOLA, 13., 2007, Rio de Janeiro. Anais ... Rio de Janeiro: SBC, 2007. Disponível em: http://www.br-ie.org/pub/index.php/wie/article/viewFile/953/939. Acesso em: julho de 2012.

CURCIO, C. P. C.. Proposta de método de robótica educacional de baixo custo. 2008. Dissertação ( Mestrado em Desenvolvimento de Tecnologia) - PRODETEC, Instituto de Tecnologia para o Desenvolvimento, Instituto de Engenharia do Paraná, Curitiba. 2008.

FILHO, D. A. M. ; GONÇALVES, P. C. Robótica Educacional de Baixo Custo: Uma Realidade para as Escolas Brasileiras. WORKSHOP SOBRE INFORMÁTICA NA ESCOLA, 2008, Rio de Janeiro. Anais ... Rio de Janeiro: SBC, 2008. Disponível em: http://www.br-ie.org/pub/index.php/wie/article/viewFile/953/939. Acesso em: julho de 2012.

KENSKI, V. M. Novas Tecnologias: o redimensionamento do espaço e do tempo e os impactos no trabalho docente. Revista Brasileira de Educação. São Paulo, n. 8, p.58 - 71, mai/jun/jul/ago, 1998.

MIRANDA, L. C. RoboFácil: especificação e implementação de artefatos de hardware e software de baixo custo para um kit de robótica educacional. 2006. Dissertação (Mestrado em Informática) - Núcleo de Computação Eletrônica, Universidade Federal do Rio de Janeiro, Rio de Janeiro, 2006.

PINTO, M. C. Aplicação de arquitetura pedagógica em curso de robótica educacional. com hardware livre. Dissertação (Mestrado em Informática) - Núcleo de Computação Eletrônica, Universidade Federal do Rio de Janeiro, Rio de Janeiro, 2011.

SCHONS, C., PRIMAZ, E. e WIRTH, G.A.P., 2004. Introdução a Robótica Educativa na Instituição Escolar para alunos do Ensino Fundamental da disciplina de Língua Espanhola através das Novas Tecnologias de Aprendizagem. Anais do I Workshop de Computação da Região Sul. 